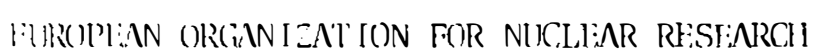

CERN-ISR-TH/80- 35

DIAGNOSTICS AND CIRES FOR BEAM INSTABILITIES

by

\begin{abstract}
A. Hofmann
Presented at XIth International Conference on High Energy Accelerators CERN, Geneva, July 7 - 11, 1980
\end{abstract}

Geneva, Switzerland July 1980 


\title{
DIAGNOSTICS AND CURES POR BEAM INSTABILITIES
}

\section{A. Hofmann}

CERN, Geneva, Switzerland

\begin{abstract}
The observation of an instability and the measurement of its properties are necessary to understand its mechanism and cause and to find means of a cure. A narrow band resonator, which drives a coupled bunch mode instability, can often be identified using the results of beam observation. Damping this resonator may then cure the instability. The transverse instabilities can furthermore be influenced by the chromaticity. The broad band impedance can be determined by observing single bunch head-tail instabilities, synchrotron and betatron frequency shifts, parasitic mode losses and bunch lengthening. A smooth wall will keep this inpedance small. Observing the response in phase and amplitude of the beam to an external excitation gives the stability diagram, the particle distribution in frequency, the wall impedance and the properties of feed-back systems. Instabilities can be cured with Landau danping provided by devices which increase frequency spread and by feed-back systems.
\end{abstract}

\section{INIRODUCTION}

The observation of the type of an instability, the measurement of its growth rate or threshold and the determination of the frequencies involved are necessary to identify an instability, to compare it with the theory and to find the cause and possible means of cures. Such measurements are also useful to check existing theories and to measure the electromagnetic properties (impedance) of the beam surroundings. The cures for instabilities can be divided into three categories: those which remove the cause or interfere directly with the mechanism of the instability, those which increase the frequency spread to provide Landau damping and feed-back system. The topics presented here have been selected with the emphasis on beam dynamics, neglecting technical and hartware developments. Only a limited number of examples have been chosen and are referred to, to illustrate certain measurements and methods. For convenience, they have often been chosen from accelerators nearby where data and pictures are easily available. In most cases the same or similar measurements have been carried out at many other machines concerned with the same effects.

\section{GENERAL METHODS AND THEORY}

Longitudinal instabilities of bunched beams manifest themselves as growing phase or energy oscillation. They can be observed as modulation (phase and amplitude) of signals from total intensity monitors or also as signals from horizontal position monitors located at a finite dispersion. For coasting beams this instability is a growing modulation of the energy and instantaneous current and can be detected on a total intensity monitor. Transverse instabilities are growing betatron oscillations and can be observed with transverse position sensitive pick-up stations. (The signal from such stations is usually proportional to the product of displacement and intensity.) Sometimes additional observations with synchrotron light monitors or with scrapers help to identify an instability. 
The theory of hunched beam instabilities is explained by J.L. Laclare in the companion (ancer ${ }^{\prime)}$. A coherent bunch oscillation can be described by two independent types of modes: the bunch shape mode describing the distortion of the bunch itself labelled by the mode number $m(m=1,2,3 \ldots$ for longitudinal dipole, quadrupole, sextupole .... modes and $m=0,1,2 \ldots$ for the transverse head-tail modes) and the coupled bunch mode describing the relative motion of different bunches with respect to each other (labelled by the mode number $n(n=M \Delta \phi / 2 \pi$; for $M$ bunches and a phase difference $\Delta \phi$ between the oscillation of adjacent bunches). The spectrun of such a mode $m, n$ consists of frequencies

$$
\begin{aligned}
& \omega_{p n}=\omega_{0}\left(p+m Q_{s}\right) \text { for the longitudinal case } \\
& \omega_{p n}=\omega_{0}\left(p+Q+m Q_{s}\right) \text { for the transverse case }
\end{aligned}
$$

with

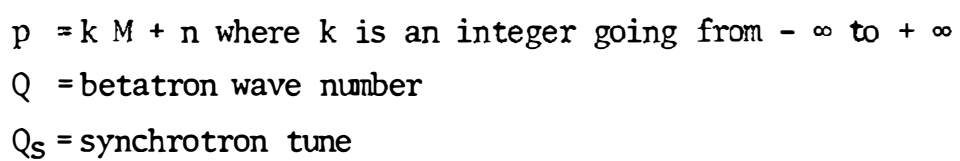

These spectral lines are shown in Fig. 1a. A coupled mode produces side bands at certain harmionics of the revolution frequency $\omega_{0}$ while a single bunch effect, where each bunch moves independently, produces side bands to each revolution hamionic. The square of the relative anplitudes of these lines $(1)$ is given by the envelope or global power spectrun $h_{m}(\omega)$ which depends on the shape node $m$, Fig. $1 b$. The horizontal scale of these spectra depends on the bunch length and the maximun of the mode $h_{1}(\omega)$ occurs at about $\omega \sim 2 \pi \mathrm{c} / \mathrm{L}$ $(\mathrm{L}=$ full bunch length). In the transverse case the spectrum is shifted due to the chromaticity $\xi=(\mathrm{dQ} / \mathrm{Q}) /(\mathrm{dp} / \mathrm{p})$ by

$$
\omega_{\xi}=\frac{\xi Q \omega_{0}}{n} ; n=\frac{1}{\gamma_{t}{ }^{2}}-\frac{1}{\gamma^{2}}
$$

The theory $1,2,3$ ) gives the conplex frequency shift $\Delta \omega_{\text {mm }}$ by which the undisturbed frequencies ( 1 ) are shifted due to the interaction between the beam and its surroundings. The real part of this shift gives the actual change of the coherent frequencies while the imaginary part gives the growth (or damping) rate of the instability. This frequency shift is calculated by multiplying the impedance with the mode spectrum and summing over all frequencies contained in the spectrum. In the longitudinal case this involves the impedance devided by the mode number $\mathrm{Z}_{\mathrm{L}}(\omega) / \mathrm{p}$; in the transverse case it is the transverse inpedance $z_{\mathrm{T}}(\omega)$. The resistive part of the impedance determines the growth rate and the reactive part the change in frequency. For a broad band inpedance and vanishing chromaticity the effect of the resistive part is small due to cancellation of the positive and negative frequency terms, Fig. Ic (a finite chromaticity disturbs this cancellation). The effects of the reactive part can, however, be large since no cancellation exists. 
a) Detailed line spectrum $\quad M=4$ coupled bunch modes

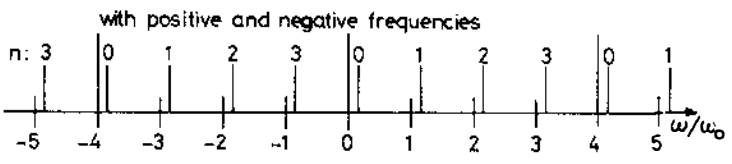

as seen on the spectrum analyser

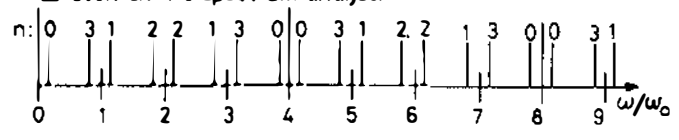

bunch shape modes

longitudina

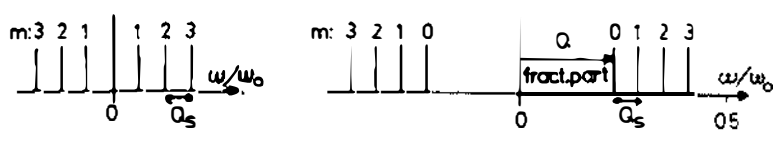

b) Global spectrum

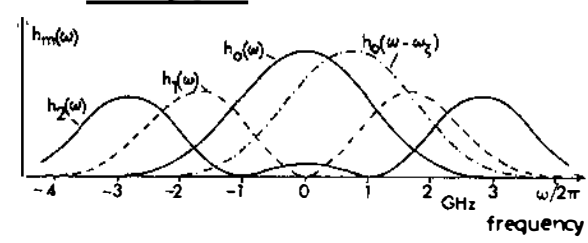

c) Brood band resonator impedonoe

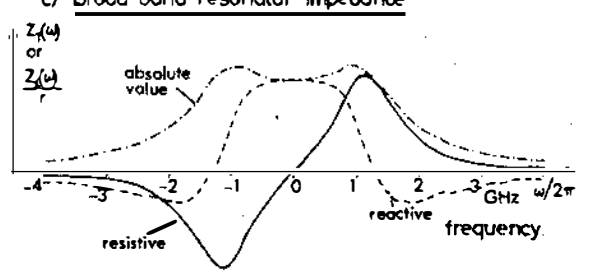

Fig. 1 Frequency spectron of longitudinal or transverse bunch oscillations and broad band resonator inpedance

3. LONGTIDUNAL COUPLED BUNCH MODE INSTABILITIES

\subsection{Observation in time domain}

For low RF-frequencies and long bunches the longitudinal oscillations can be observed directly on an oscilloscope. The period of oscillation and the relative phase between adjacent bunches (node $\mathrm{n}$ ) is best measured with a "mountain range" display, Fig. 2 4). The growth rate is obtained by measuring the amplitude of oscillation as a function of time which can be done on a multiple sweep "mountain range" display, Fig. 3 4). The bunch shape mode $\mathrm{m}$ is obtained from the measured period length or from a superimposed (multiple sweep) picture where $m$ is equal to the number of nodes ${ }^{5}$ ).

\subsection{Observation in frequency domain}

For high RF-frequencies and short bunches it is difficult to see longitudinal oscillations on an oscilloscope and observation in frequency domain (spectnm analyser) is more appropriate. The couple bunch mode number $\mathrm{n}$ can be determined by observing which revolution harmonics show upper or lower side bands. The appearance of all side bands indicates a single bunch effect. In Fig. 4 the spectrun of five bunches accelerated in the CERN-PS 
ixuster is shown as a "mountain range" display"). The extreme lines show the RF-frequency (which thanges during accolcration) and its first harmonic. The side bands of the 7 th and sth rovolution hamonic grow with time, indicating the growth rate of the instability. With a better resolution it could be shown that the 7 th harmonic has a lower side band and the 8 th harmonic an upper one, which determines the coupled mode number $\mathrm{n}=3$.

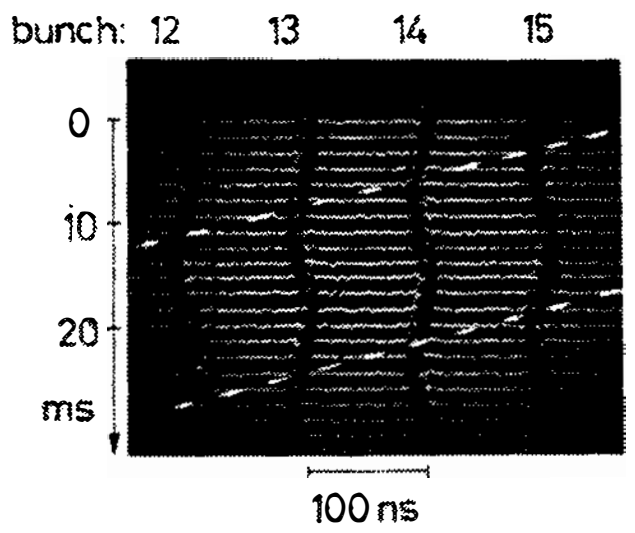

Fig. 2 Phase relation between the oscillations of adjacent bunches (mode $n$ ) seen on a "moun tain range" display ${ }^{4}$ )

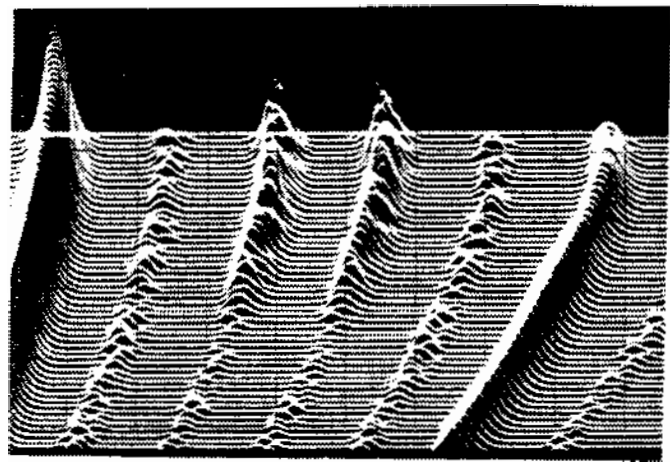

Fig. 4 "Mountain range" display of the spectrum from 5 bunches during acceleration in the CERN-PS Booster ${ }^{6}$ )

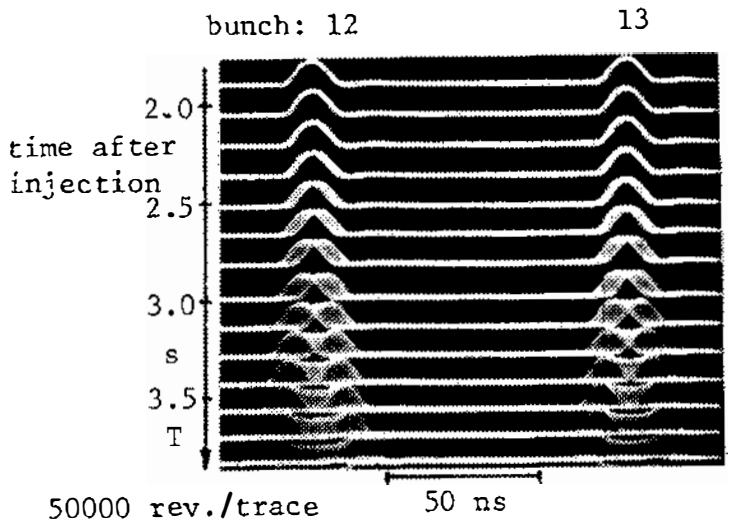

Fig. 3 Growth rate of a dipole instability seen on a multiple sweep "mountain range" display ${ }^{4}$

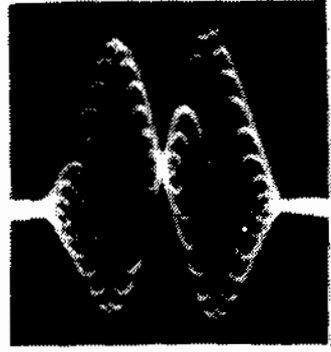

$m=1$

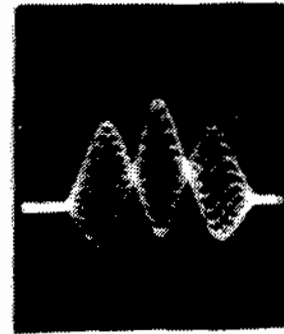

$n=2$
Fig. 5 Multi-sweep display of the headtail modes $m=1$ and $m=2$ of $a$ single bunch ${ }^{10}$ )

\subsection{Application}

From the instability observation the coupled mode number $n$ and the shape mode number $m$ can be determined. This is often sufficient to design a feed-back system or another device to stabilize the particular mode"). However, it is preferable to find the offending element which drives the mode and cure the instability at the source. To do this, the properties of this resonator have to be determined. The knowledge of the mode number $n$ is not sufficient to determine the frequency of the resonator according to Fig. 1a. This ambiguity is specially pronounced in a machine with only few bunches. Filling all buckets in such a machine will of course help to find the resonator frequency, and for a coasting beam the ambiguity disappears. Additional information can be obtained by observing the modes $\mathrm{m}$ for 
different bunch lengths. Observing the growth rate of the instability for different bunch spacings gives some information of the decay time of the induced fields (quality factor of the resonator). The growth rate itself gives the impedance ${ }^{4}$ ). Based on this information, some elements might be suspected. If they contain coupling loops (as is often the case for cavities), the fields can be measured directly while the instability occurs $\left.{ }^{8}, 9\right)$. After identification, the dangerous modes in the offending element should be damped with resistors or terminated coupling loops.

\section{TRANSVERSE INSTABIL ITIES}

Apart from the effect of the chromaticity the transverse corpled bunch mode instabilities behave very similarly to the longitudinal ones. They can be analysed using the methods described in the last chapter in order to find the offending element ${ }^{11}$ ). The bunch shape modes are influenced by the chromaticity which makes the head and the tail of a bunch oscillate with a different phase. This inposes the characteristic wiggle on the bunch, shown in time domain in Fig. $S^{10}$ ), and produces the shift $\Delta w_{\xi}$ of the global spectrum shown in Fig. $1 b$. The bunch shape mode number m can directly be determined as the number of nodes on a multiple sweep display, like the one shown in Fig. 5. For non-vanishing chromaticity $\xi$ a single bunch head-tail instability can occur. Above transition energy and for normal conditions the lowest mode $m=0$ is stable for a positive chromaticity while some of the higher modes (depending on the bunch length and the form of the inpedance) are stable for negative $\xi$. Coupled bunch mode head-tail instabilities can, however, occur even for $\xi=0$.

The observation of a head-tail instability is illustrated on Fig. 6 12). The loss of bunches is observed on a total intensity monitor signal (top). The vertical position monitor signal (bottom) is propoertional to displacement times intensity and growth with the instability to decay again when the bunch gets lost. The global spectrum is shown in Fig. 7 12) and agrees well with the calculated spectrum for the mode $m=0$ using the appropriate bunch length and chromaticity frequency shift.

The head-tail instability can be used to measure resistive part of the transverse wall inpedance of a machine. The instability probes the impedance with the spectrum $h_{1}(\omega-\omega \xi)$ shown in Fig. 7 which can be moved by changing $\xi$. Such a measurement has been done for the CERN-PS ${ }^{10}$ ). The result is plotted in Fig. 8 as the equivalent longitudinal inpedance $\mathrm{z}_{\mathrm{L}}(\omega)$. Instead of using the instability, the head-tail effect can also be investigated by using positive chromaticity and observing the damping of excited coherent oscillations ${ }^{13}$ ). The real betatron frequency shift of a head-tail mode due to the reactive impedance has been measured at $\mathrm{DCI}^{15}$ ) and at PETRA ${ }^{16}$ ). The results shown in Fig. 9 show the shift of the first two or three modes in DCI and indicate a relatively large shift for large machines with few short bunches like PEIRA.

The transverse coupled bunch mode instability driven by a narrow band resonator can be stabilized by providing head-tail damping of the single bunch modes due to the broad band impedance. For the lowest mode $m=0$ (above transition energy) this is achieved by choosing a chromaticity which is not zero but slightly positive ${ }^{17}$ ). Some higher modes could then become unstable, but they are usually mach weaker. 

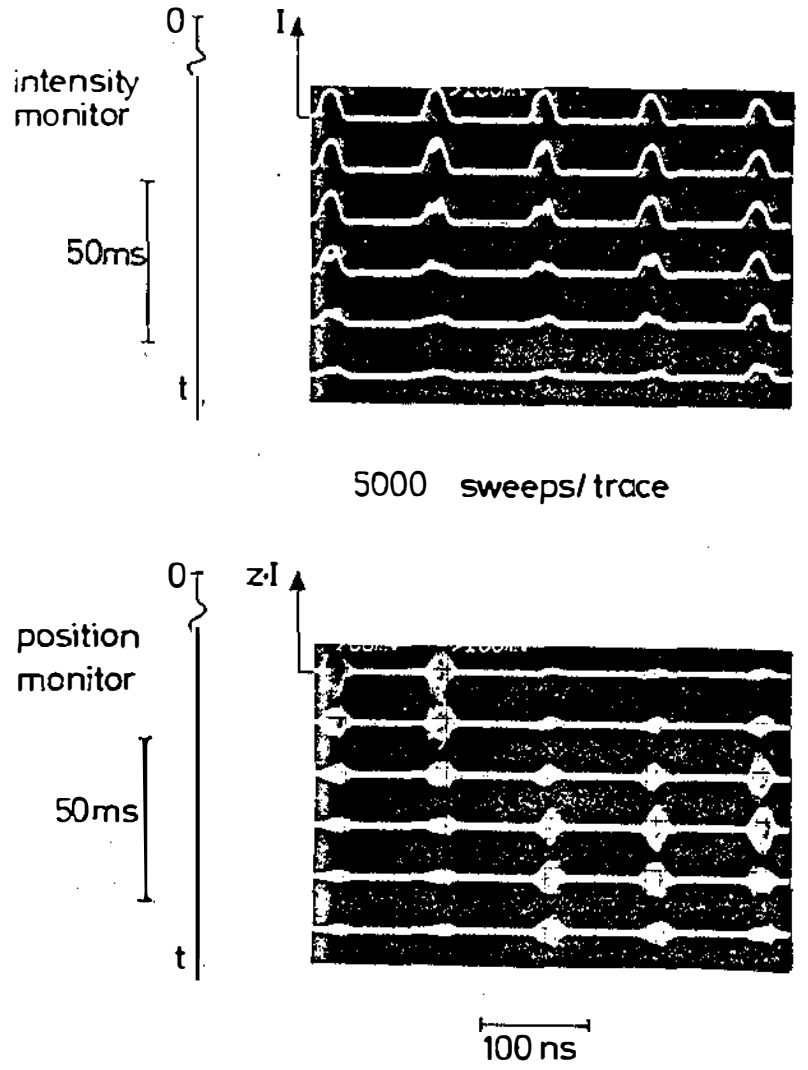

Fig. 6 Observation of a head-tail instability using a "mountain range" display of the signals from the total intensity and from the position sensitive monitor ${ }^{2}$ ).
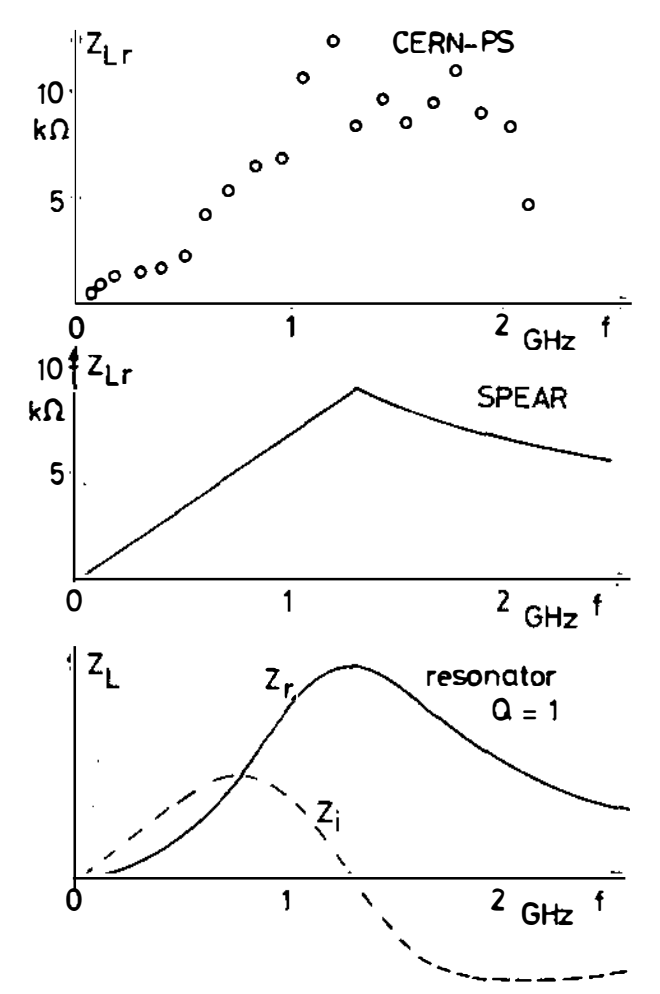

Fig. 8 Impendace measurements. Top: measured with head-tail instability in the CERN-PS ${ }^{10}$. Middle: obtained from parasitic mode losses and bunch lengthening in SPEAR ${ }^{14}$. Bottom: broad band resonator impedance for conparison.
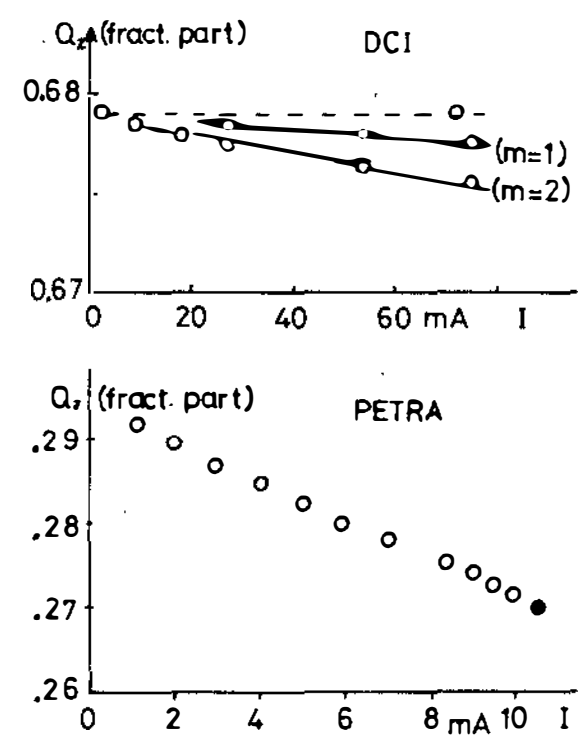

Fig. 9 Betatron frequency shift of the head-tai modes as a function of current in DCI ${ }^{15}$ ) and PETRA ${ }^{16}$ ). 


\section{LONGITUDINAL SINGLE BUNCH EFFECTS}

\subsection{Parasitic mode losses}

The currents induced by the bunch in the resistive wall lead to an energy loss called parasitic mode loss. This is not an actual instability but an effect which can be annoying in large machines with few high intensity bunches. This loss is given by the product hetween the power spectrum $h_{0}(\omega)$ of the stationary bunch distribution and the resistive 1ongitudinal impedance $Z_{\operatorname{Lr}}(\omega)$ integrated over all frequencies ${ }^{14}$ ). The effect can be used to estimate the wall impedance. It is measured by observing the shift of the synchronous RFphase angle with beam current necessary to compensate the increased energy loss ${ }^{16}$ ).

\subsection{Synchrotron frequency shift and potential well bunch lengthening}

The beam-induced fields in the longitudinal reactive impedance lead to a distortion of the RF-wave form seen by the particles inside the bunch. This can lead to a lengthening and distortion of the bunch and to a charge of the synchrotron frequencies. Long bunches probe the wall at low frequencies where the impedance is predominantly inductive. In this case a bunch with parabolic line density in a linear RF-wave forn represents a selfconsistent distribution suffering only potential well lengthening without distortion. The incoherent synchrotron frequencies and the coherent frequencies of the higher modes (m 2 2) are reduced. The coherent dipole mode $(m=1)$ is not changed because the potential well moves with the bunch. The effect is illustrated in Fig. 10 where the decrease of the quadrunole mode frequency with current is plotted for the $I S R^{18}$ ), indicating an inductive impedance $\mathrm{Z}_{\mathrm{Li}} / \mathrm{p} \sim 20 \mathrm{Ohm}$ at low frequencies. The increase of the bunch length is shown in Fig. 11 19). It agrees quite well with the theoretical prediction up to the threshold of turbulence which will be discussed later. The bunch area shown at the bottom of Fig. 11 is expected to stay constant for potential well bunch lenghtening of protons. For short bunches which probe a more complicated impedance and for different bunch forms the effect is more complex. The bunch suffers a distortion and the frequency shifts are more difficult to interpret.

\subsection{Microwave instabilities (turbulence)}

Above a certain threshold current a blow-up of the bunch in length and energy spread occurs connected with the appearance of high frequency signals ${ }^{20}$ ). The occurring increase of the bunch length with current has been measured in many machines. Observing also the increase of energy spread $\left.{ }^{14}, 17,21\right)$ clearly distinguishes the turbulent bunch lengthening from the effects of the potential well. This is illustrated by the measurement shown in Fig. 11 where potential well bunch lengthening is observed at low currents and additional turbulent lengthening at higher currents. The turbulent bunch lengthening data measured in different machines can be described relatively well with the coasting beam mode1 ${ }^{22}$ ). It applies the longitudinal stability criterion ${ }^{23}$ ) for coasting (unbunched) beams to the peak current $I_{p}$ and rms energy spread $\sigma_{E} / E$ in the bunch and gets for the equilibrium condition abvoe the turbulent threshold 


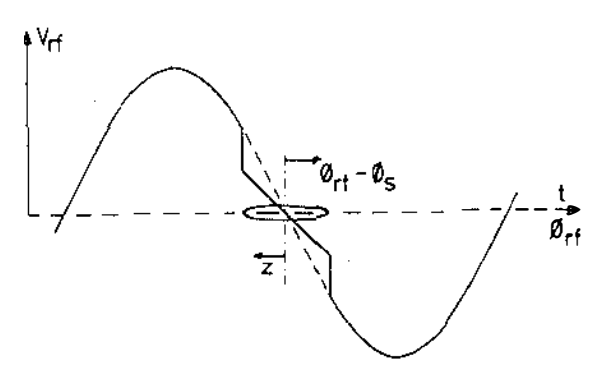

$R F$ - voltage with inductive wall

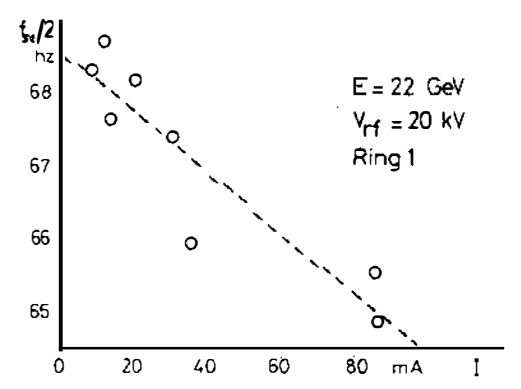

Synchrotron frequency vs. beam current

Fig. 10 Potential well due to an inductive impedance and resulting shift of the quadrupole mode synchrotron frequency ${ }^{18}$ ).

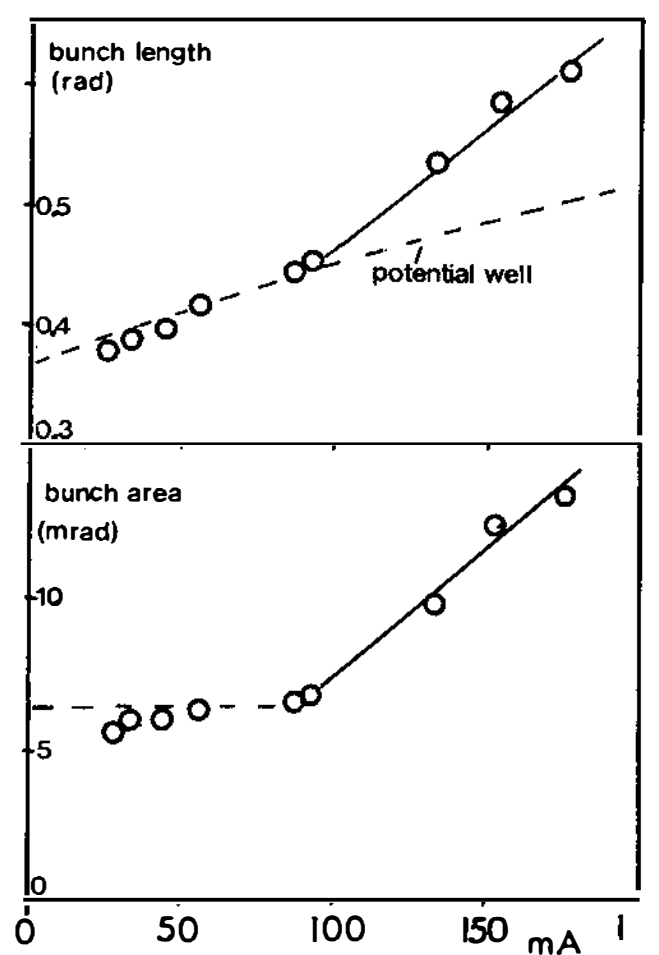

Fig. 11 Potential well and turbulent bunch lengthening for protons ${ }^{19}$ ).

$$
\left|\frac{z}{p}\right|_{\text {crit }} \approx \frac{F^{\prime} E \cdot|n|}{e} \cdot \frac{\left(\sigma_{E} / E\right)^{2}}{I_{p}}
$$

with $F^{\prime}$ being a form factor or the order of 6 . 'This can be rewritten to get the equilibrium rns bunch length $\sigma_{S}$ in a machine of radius $R$ with an average current $I_{0}$ in $M$ bunches.

$$
\left(\frac{\sigma}{\bar{R}}\right)^{3} \approx \frac{\sqrt{2 \pi}}{\mathrm{F}^{\top}} \frac{\mathrm{e}}{\frac{|n| \mathrm{I}_{\mathrm{O}}}{\mathrm{M}}} \frac{\mathrm{Z}}{\left.\overline{\mathrm{P}}\right|_{\text {crit. }}{ }^{2} \mathrm{E}}
$$

Long bunches probe the inpedance at low frequencies where $|z / p|$ crit is approximately constant (see Fig.8) and the bunch length scales about as $\sigma_{S} \propto I_{0}{ }^{1 / 3}$. This is observed for proton bunches and long electron bunches. Short bunches probe the impedance at high frequencies where $|z / p|_{\text {crit }}$ depends on frequency and therefore on the bunch length. The latter is expected to show a stronger dependance on the current in agreement with observations made on short electron bunches ${ }^{34}$. For electrons the observed blow-up affects mostly the core and less the tails of the bunch as qualitatively expected from some aspects of the coasting beam mode $1^{24}$ ). More sophisticated theories have been developed to explain turbulent bunch lengthening like the mode coupling theory ${ }^{1,3}$ ), but comparison between the predictions and the observations is more complicated.

To cure or improve the longitudinal single bunch phemonema the broad band impedance 
should be reduced by making the chamber wall smooth. I)istributing the current into morc bunches and increasing the bunch length also improve the situation. The microwave instability for protons can furthernore be improved according to (2) by using bunches with large energy spread ${ }^{19}$ ).

\section{BEAM TRANSFER FUNCTION}

The response in amplitude and phase of a beam to an external excitation contains a great deal of information about the beam stability, the wall impedance and the particle distribution in the beam ${ }^{25-29}$ ). This response is often called beam transfer function in analogy to its use in network analysis. It can be obtained by measuring the response to a swept frequency sinosoidal excitation using a network analyser or to white noise excitation using a fast Fourier transform analyser (FFI). For linear systems the two methods are equivalent.

The beam transfer function can be illustrated on the simple case of a transverse excitation with frequency $\omega$ of a coasting beam with revolution frequency $\Omega$ and betatron tune $Q$ (both being functions of the momentum deviation only). The equation of motion of a single particle is

$$
\ddot{x}+Q^{2} \Omega^{2} x=G \exp (-i \omega t) .
$$

For coasting beams solutions of the form $x=x_{0} \exp [i(p \theta-\omega t)]$ are appropriate where $\theta$ is the betatron phase advance between exciter and monitor divided by $Q$. The relative response becomes :

$$
\frac{x_{0}}{G}=\frac{-\exp (-i p \theta)}{[\omega-\Omega(p+Q)]} \frac{[\omega-\Omega(p-Q)]}{[\omega}
$$

It is only large if the exciting frequency $\omega$ is close to $\omega_{\beta}=\Omega(p+Q)$, (fast wave) or $\omega_{B}=\Omega(p-Q)$, (slow wave). Taking the second case and assuming $\theta=0$ the response of the center of charge is obtained by averaging (5) over the distribution $F\left(\omega_{B}\right)$ of the $N$ particles in betatron frequencies $\omega_{\beta}$.

$$
\left\langle\frac{x}{G}\right\rangle=\frac{I}{2 \Omega_{0} Q_{0} N} \int \frac{F\left(\omega_{B}\right) d \omega_{B}}{\omega-\omega_{B}}=\frac{1}{2 \Omega_{0} Q_{0} N}[P V+i \pi F(\omega)]
$$

with $\Omega_{0}$ and $Q_{0}$ being the values at the center of the distribution. This dispersion integral has a principle value PV and a residue. The velocity response $\dot{x}$ to the acceleration $G$ becomes :

$$
\left\langle\frac{\dot{x}}{G}\right\rangle=\frac{\omega}{2 \Omega_{0} Q_{O} N}[\pi F(\omega)+i p V] .
$$

It has a real part of the response being in phase with the excitation which absorbs energy and provides Landau damping. This term gives directly the particle distribution in incoherent betatron frequencies and vanishes if the exciting frequency $\omega$ is outside this distribution. The inverse of the response (7) is the well known stability diagram which can be measured this way.

The fields induced in the wall impedance produce an additional force on the beam which has to be added to the external excitation in (4). This leads to a shift of the 
misured nomalized stahility diagram in the direction opposite to the wall impedance by an amount which is proportional to the impedance times current divided by the frequency spread. This is illustrated by the measurement shown in Fig.12 where the transfer function of a coasting bcam has been measured for different frequency spreads (obtained by changing the chromaticity $\xi=Q^{\prime} /($ ). It shows an increase of the beam response and a shift of the stability diagram with decreasing frequency spread. Such a measurement tells how stable the beam is, that is, how much the current could be increased or the frequency spread reduced before an instability occurs. It also gives the real and imaginary part of the wall impedance. Applications of the transfer functions are described in contributions to this conference $30-33)$.

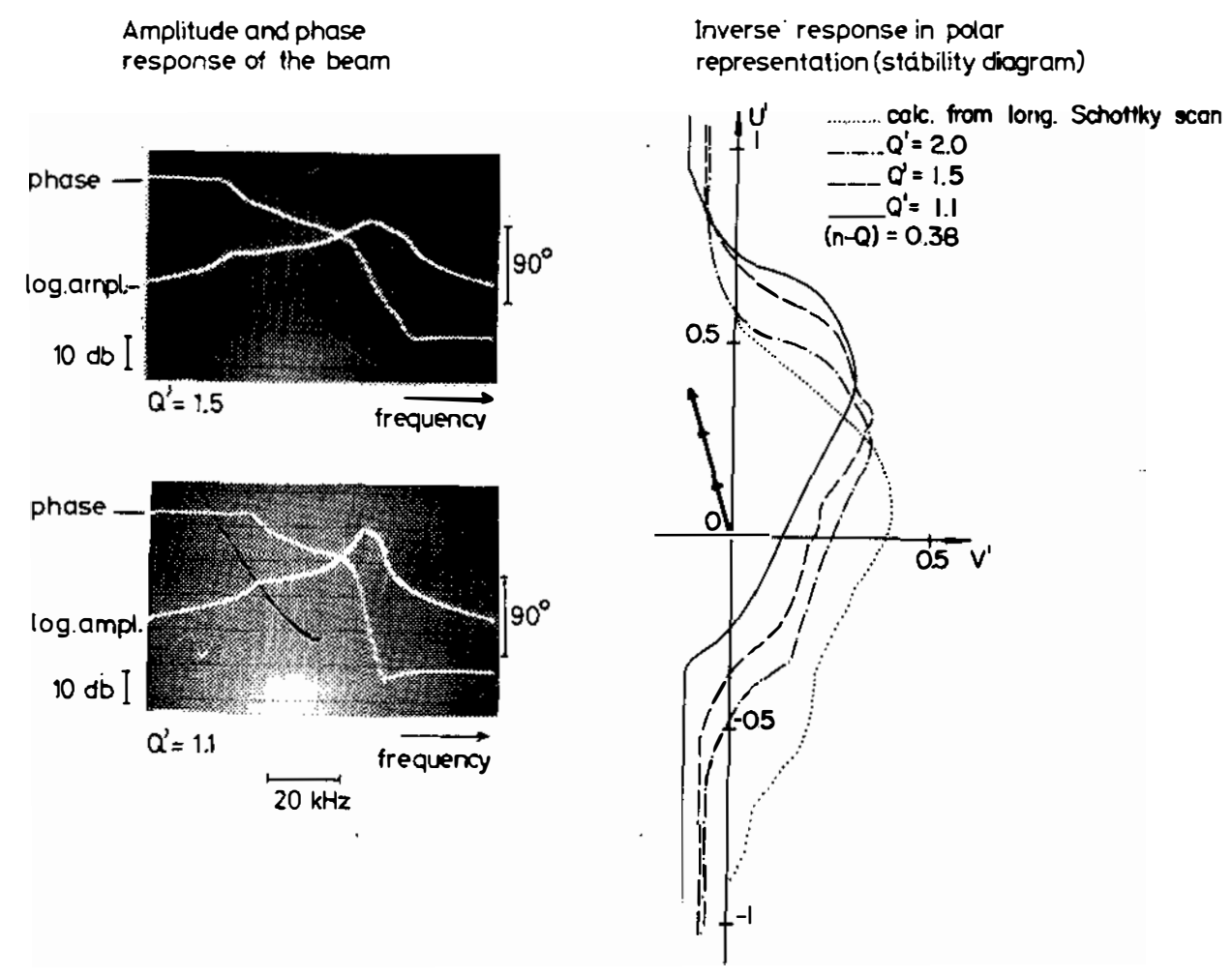

Fig. 12 Measurement of the beam transfer function ${ }^{28}$ )

\section{REMARKS ON IMPEDANCES}

In order to compare the observed instabilities with the theory the wall inpedance should be known. For the coupled bunch mode instabilities the parameters of the driving resonator is often known and good agreement between calculated and measured growth rates is usually found ${ }^{4}$. Single bumch effects are due to a broad band inpedance which may consist of many single resonances not resolved by the effect. This inpedance is usually not well known but seems to have some common featires in different machines. The measurements shown 
in Fig. 8 indicate that the resistive part rises first with frequency reaches a maximum somewhere around the cut-off frequency where wave propagation in the chanther hecomes possible and decays slowly at higher frequencies. It is often convenient to approximate this behaviour by an inpedance of a broad band resonator which has simple expressions for the resistive and reactive part. The absolute value of the longitudinal impedance divided by the mode number $\left|z_{L} / p\right|$ at low frequency has become a convenient measure for the hroad hand impedance. It does not increase with the size of the machine but denends only on the installed impedance per unit length and lies between 10-30 Ohms for classical machines but is much smaller 1-2 Ohms for a smooth vacuun chamber. The transverse broad band impedance $z_{\mathrm{T}}$ can he estimated from the longitudinal one using the approximate relation

$$
\mathrm{z}_{\mathrm{T}}(\omega) \approx \frac{2 \mathrm{R}}{\mathrm{b}^{2}} \frac{\mathrm{z}_{\mathrm{L}}(\omega)}{\mathrm{p}}
$$

where $R$ is the radius of the machine and $b$ a suitable effective chamber radius. This relation tends to overestimate $z_{\mathrm{T}}$ but is still usefull to relate longitudinal and transverse observationswith each other. Using such simple pictures one finds reasonable agreement between theory and observation of single bunch phenomena. In the case of turbulence some more detailed measurements might be necessary to check the mode coupling theory. The impedance is usually not known well enough to check higher head-tail modes.

The single bunch frequency shifts for the head-tail modes and the longitudinal modes often separate the coherent frequencies from the incoherent distribution and lead to a loss of Landau damping.

\section{CTRES FOR INSTABILITIES}

\subsection{RF-quadrupoles and $Q_{s}$-splitting cavities}

A transverse or longitudinal coupled bunch mode instability can be reduced with a quadrupole or a cavity which oscillates at a frequency which is a harmonic of the revolution frequency but not of the bunch frequency ${ }^{7}, 8,17$ ). This provides different transverse or longitudinal focusing for different bunches and results in a spread of the betatron or synchrotron frequency from bunch to bunch. The bunches, or groups of bunches, are decoupled from each other and the instability is reduced. The choice of the operating frequency depends on the memory of the impedance which could drive the instability. A frequency of the order of inverse decay time of the induced fields is most effective. If the $n_{s}$-splitting cavity has a high impedance it could drive an instability itself unless it operates at half the bunch frequency. This and practical considerations influence the choice of the parameters of such devices.

\subsection{Higher harmonic cavities}

Such a cavity operates at a harmonic of the bunch frequency and increases the nonlinearity of the phase focusing force. This results in an amplitude dependence of the synchrotron frequency and results in frequency spreadnecessary for Landau damping. Such a cavity can be operated such as to increase the slope and non linearity of the normal RFwave form. In this mode a passive cavity can be used driven by the beam ${ }^{4}, 17$ ). It is most effective to operate a higher harmonic cavity in the bunch lengthening mode where it reduces 
the shope of the totil kl-wave form to zero in the bunch center. The synchrotron frequency hcrmus zro for vanishing armplitudes and increases about linearly with amplitude to level ofl later. The synchrotron frequency spread is very. large and the resulting Landau damping can he very strong. The phase motion is non-linear having ahout $4.5 \% 3$ rd harmonic content ${ }^{34}$. The bunch is lengthened and the peak current reduced. The effect of such a ciavity on a single beam is quite impressive. All longitudinal instabilities disappear and longitudinal oscillations excited by a kick hecome incoherent in a very short time indicating the strong Landau clamping. The operation of such a cavity is indicated in Fig.13 19). Such a cavity might also provide some Landau damping for higher head-tail modes which involve the synchrotron frequency (1). Some difficulties have been encountered at SPEAR ${ }^{35}$ for operating such a cavity in a colliding beam mode close to the beam-beam liunit.
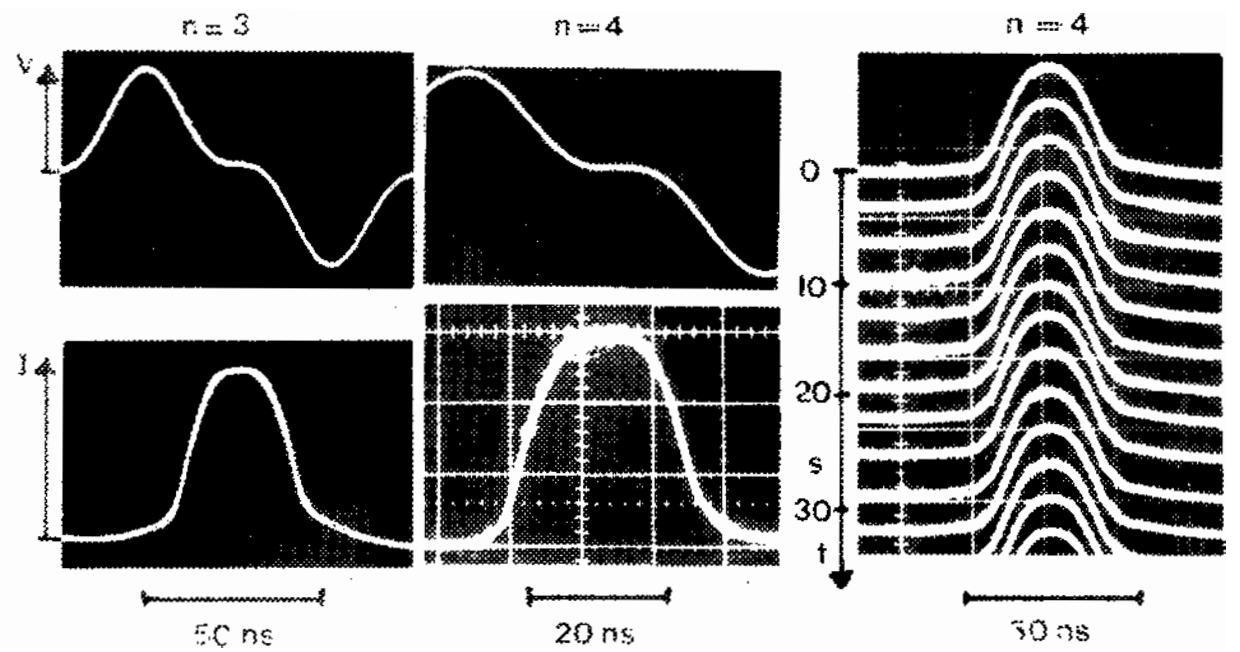

Fig. 13 Wave form, bunch shape and bunch stability for operation with a thirci and fourth harmonic cavity ${ }^{29}$ ).

\subsection{Feed-back systems}

Feed-back systems are devices which sense the deviation of the beam and take corrective action. Practically all machines use one or several feed-back systems for operation. The discussion presented here is restricted to some beam dy namical aspects related to the theory outlined in the companion paper ${ }^{3}$ ).

A feed-back system senses the oscillation of the bunches and takes a corrective action a quarter (or $3 / 4,5 / 4 \ldots$ ) of an oscillation later. Translated into frequency domain, as illustrated in Fig. 1 , this action is equivalent with having an impedance $\left(z_{\mathrm{L}}(\omega) / p\right.$ or $\left.z_{\mathrm{T}}(\omega)\right)$ which has a positive resistive part for positive and negative frenuencies. Using positive frequencies only this can be expressed as having a positive resistive impedance at the upper side band and a negative one at the lower one ${ }^{36}$. In the longitudinal case the signs of this impedance has to be changed above transition energy. The feed-back system can then be 
treated with the same theory as the instability. In order to stabilize all coupled morles $n$ the band width of the feed-back has to be as large as half the bunch frequcncy; Fig. 1a. To stabilize more than one of the shape modes $m$ this frequency hand has to lie in a region where the global spectra $h_{m}(\omega)$ are not too small.

This action of a transverse feed-back system is illustraded in Fig. 14 37). On the left side the transfer function of the fast and slow wave is shown without the feed-back system. The stability diagram is shifted by the vector -2 ' due to the predominantly inductive wall impedance. The effect of feed-back system is shown on the right side where the two diagrams are moved in opposite direction enlarging the stable region. By having a phase shift between monitor and kicker different than $90^{\circ}$ a feed back system can counteract a complex impedance shifting the stability diagram back to the origin ${ }^{3}$ ). This could act against frequency shifts and reinsta11 Landau damping.

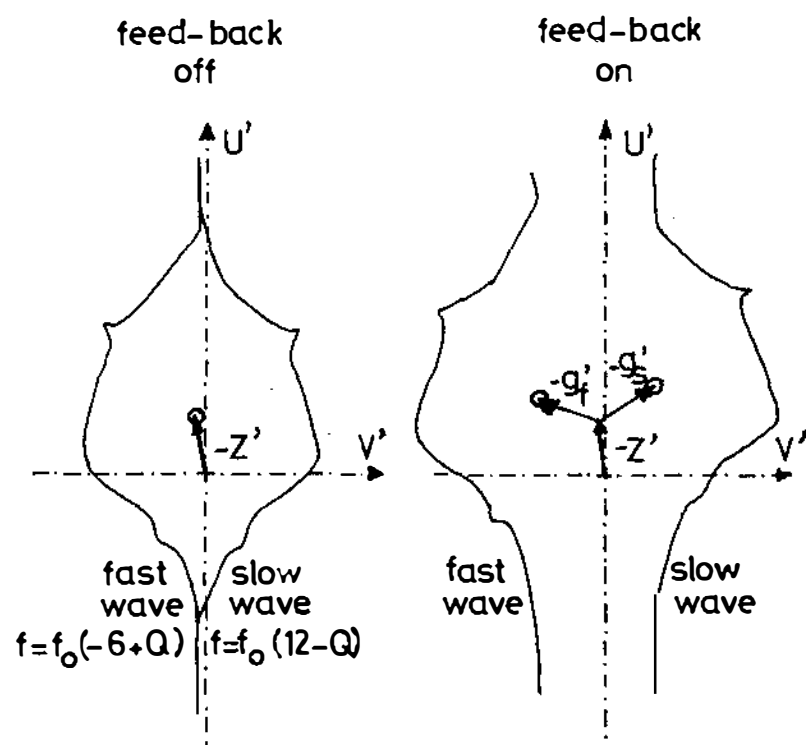

Fig. 14 Action of a transverse feed-back system demonstrated by the beam transfer function ${ }^{37}$ ).

\section{REFERENCES}

1) J.L. Laclare, invited paper at this conference.

2). F. Sacherer, 9th Int. Conf. on High Energy Acce1., Stanford 1974, p.347.

3) F. Sacherer, IEEE Trans. on Nuc1. Sci. NS-24, p.1393 (1977).

4) P. Bramham et a1, 9th Int. Conf. on High Energy Acce1., Stanford 1974, p.359.

5) F. Pedersen, F. Sacherer, IEEE Trans, on Nuc1. Sci. NS-24, p.1396 (1977).

6) J. Gareyte et a1, IEEE Trans. on Nuc1. Sci., NS-22, p.1855 (1975).

7) C.M. Ankenbrandt et a1, IEEE Trans. on Nuc1. Sci. NS-24, p.1449 (1977).

8) R.D. Kohaupt, IEEE Trans. on Nuc1. Sci., NS-22, p.1456 (1975).

9) D. Boussard et a1, IEEE Trans. on Nuc1. Sci., NS-24, p.1399 (1977). 
10) J. (iareytc, F. Sacherer, 9th Int. Conf. on High Energy Acce1., Stanford 1974, p.341.

11) Y. Miyahara, IElEE Trans. on Nucl. Sci., NS-26, p.3535 (1979).

12) A. llofmann, F. Sacherer, ISR Perf. Rep. Runs 669-908 (1978).

13) The SPEAR Group, 9th Int. Conf. on High Energy Accel., Stanford 1974, p.338.

14) P.B. Wilson et a1, IEEE Trans. Nucl. Sci., NS-24, p.1211 (1977).

15) M.P. Level, Orsay LAL-RT-79/5.

16) R.D. Kohaupt, IEEE Trans. on Nucl. Sci. NS-26, p.3480 (1979).

17) "R. Averill et a1, 8th Int. Conf. on High Energy Accel., CERN 1971, p.301.

18) S. Hansen et al, IEEE Trans. on Nuc1. Sci. NS-22, p.1381 (1975).

19) P. Bramham et al, IEEE Trans. on Nucl. Sci. NS-24, p.1490 (1977).

20) W. Schne11, IEEE Trans. on Nucl. Sci. NS-22, p.1357 (1975).

21) The Adone Group, IEEE Trans. on Nucl. Sci., NS-18, p.217 (1971).

22) D. Boussard, CERN Lab II/RF/int./75-2 (1975).

23) E. Keil, W. Schne11, CERN-ISR-TH-RF/69-48 (1969).

24) A. Hofmann, F. Pedersen, IEEE Trans. on Nucl. Sci., NS-26, p.3526 (1979).

25) H. Grunder, G. Lambertson, 8th Int. Conf. on High Energy Accel. CERN 1971, p.308.

26) D. Möhl, A. Sessler, 8th Int. Conf. on High Energy Accel., CERN 1971, p.334.

27) A. Faltens et al, Int. Conf. on High Energy Accel., CERN 1971, p.338.

28) A. Hofmann, B. Zotter, IEEE Trans, on Nucl. Sci., NS-24, p.1478 (1977).

29) J. Borer et al, IEEE Trans. on Nucl. Sci., NS-26, p.3405 (1979).

30) J. Borer et a1, Contribution to this conference.

31) J.Y. Hemery et al, Contribution to this conference.

32) J.P. Koutchouk, Contribution to this conference.

33) E. Peschardt, Contribution to this conference.

34) A. Hofinann, S. Myers, Contribution to this conference.

35) M.H.R. Donald, J.M. Paterson, IEEE Trans. on Nucl. Sci., NS-26, p.3580 (1979).

36) F. Pedersen, F. Sacherer, IEEE Trans. on Nucl. Sci., NS-24, p.1396 (1977).

37) J.C. Juillard, E. Peschardt, ISR Perf. Rep., Aug. 1979. 